\title{
ICAM- 1 and VCAM- 1 antisense oligonucleotides attenuate in vivo leucocyte adherence and inflammation in rat inflammatory bowel disease
}

\author{
E Rijcken, C F Krieglstein, C Anthoni, M G Laukoetter, R Mennigen, H U Spiegel, \\ N Senninger, C F Bennett, G Schuermann
}

See end of article for authors' affiliations

Correspondence to: Dr C F Krieglstein, Department of General Surgery, Westphalian Wilhelms-University, Waldeyerstr 1, D-48149 Muenster, Germany;

krieglstein@uni-muenster.de

Accepted for publication 28 February 2002

\begin{abstract}
Background: Recruitment of circulating cells to the inflamed intestine is modulated by adhesion molecules expressed on the surface of both leucocytes and endothelial cells.

Aims: The objective of this study was to test whether 2'-O-methoxyethyl chimeric antisense oligonucleotides directed against endothelial intercellular adhesion molecule 1 (ICAM-1) and vascular cell adhesion molecule 1 (VCAM-1) can downregulate leucocyte-endothelial interactions and thereby attenuate inflammation in rat experimental ileitis.

Methods: Indomethacin $(7.5 \mathrm{mg} / \mathrm{kg}$ ) was injected subcutaneously into Sprague-Dawley rats 48 and 24 hours prior to intravital microscopy. Animals were treated with either ICAM-1 (ISIS 17470), VCAM-1 (ISIS 18155), or scrambled control antisense oligonucleotides administered subcutaneously or intravenously in parallel with indomethacin. Leucocyte trafficking was observed in ileal submucosal collecting venules. Macroscopic and histological grades of inflammation were measured 48 hours after the first indomethacin application. ICAM-1 and VCAM-1 expression in ileal submucosal venules was detected by immunohistochemistry.

Results: Intravenous administration of ICAM-1 oligonucleotides 2 mg/kg (rolling leucocytes 5.7 (2.4)/ $0.01 \mathrm{~mm}^{2}$ endothelial surface, adherent leucocytes $\left.0.8(1.1)\right)$ and VCAM-1 oligonucleotides $8 \mathrm{mg} / \mathrm{kg}$ (9.2 (4.4), $0.6(0.8))$ significantly reduced leucocyte adhesion compared with diseased controls (27.8 (5.3), 14 (4.4)) in a dose dependent manner whereas subcutaneous treatment did not. Correspondingly, macroscopic and histological inflammation was significantly decreased. ICAM-1 oligonucleotides markedly reduced endothelial ICAM-1 expression while VCAM-1 oligonucleotides clearly diminished endothelial VCAM-1 expression.

Conclusions: Both ICAM-1 and VCAM-1 2'-O-methoxyethyl chimeric antisense oligonucleotides attenuate rat ileitis by downregulation of leucocyte adherence and thus are potential candidates for anti-inflammatory treatment in inflammatory bowel disease.
\end{abstract}

l: $\mathrm{n}$ inflammatory bowel disease (IBD) there is a dense intestinal infiltrate of inflammatory cells which exhibit a distinct distribution for Crohn's disease as well as ulcerative colitis. For the development of the local intestinal cellular infiltrate, circulating cells must adhere to the intestinal vascular endothelium and transmigrate into the tissue where the immuno-inflammatory reaction is created. A multistep cascade for adhesion of circulating leucocytes to endothelial cells has been proposed, involving specific families of adhesion molecules which are expressed on endothelial cells and leucocytes as ligands and counterparts. ${ }^{1-3}$ Intercellular adhesion molecule (ICAM)-1 belongs to the immunoglobulin superfamily and is constitutively expressed not only on endothelial cells but also on lymphocytes, monocytes, epithelial cells, and other cells. It can be upregulated transcriptionally by endotoxin or by proinflammatory cytokines such as tumour necrosis factor (TNF)- $\alpha$ and interleukin (IL)- $1 .{ }^{45}$ Increased expression of endothelial ICAM-1 in inflamed tissues promotes the recruitment of inflammatory cells expressing its ligands leucocyte function antigen 1 (LFA-1) (CD1la/CD18) or MAC-1 (CD1lb/CD18). ${ }^{6}$ Likewise, vascular cell adhesion molecule (VCAM)-1 belongs to the immunoglobulin superfamily and is expressed on activated endothelial cells, macrophages, dendritic cells, and fibroblasts. While binding to its ligands integrin $\alpha 4 / \beta 4$ (very late antigen 4 (VLA-4)) and $\alpha 4 / \beta 7$ on lymphocytes, monocytes, and eosinophils, VCAM- 1 modulates leucocyte firm adhesion to endothelial cells and migration to sites of inflammation. ${ }^{23}$ Despite former assump- tions, integrin $\alpha 4$ has also been shown to be expressed on the surface of neutrophils in rats, mice, and humans. ${ }^{7-10}$ There is increasing evidence that leucocytes can also directly tether, roll, and adhere to the endothelium under inflammatory conditions using the $\alpha 4$ integrin alone which may represent an alternative pathway to the established model of leucocyte adhesion. ${ }^{89}$ Upregulation of ICAM-1 has been shown in actively inflamed mucosa in IBD, mostly by means of immunohistochemistry, ${ }^{11-13}$ corresponding to elevated serum levels of both ICAM-1 and VCAM-1 in active disease. ${ }^{14}{ }^{15}$ Only a few studies have failed to demonstrate increased ICAM-1 or VCAM-1 expression ${ }^{12} 1{ }^{16}{ }^{17}$ in human IBD tissue. However, in cell cultures ${ }^{5}$ and animal models ${ }^{18} 19$ ICAM-1 and VCAM-1 were markedly increased under inflammatory conditions. Therefore, reduction of ICAM-1 and VCAM-1 expression may elicit potent anti-inflammatory activity.

Synthetic phosphorothioate antisense oligonucleotides have been shown to block expression of specific targeted proteins by arrest of translation, inhibition of processing, or degradation of the targeted mRNA. ${ }^{20-22}$ Antisense oligonucleotides

Abbreviations: FITC, fluorescein isothiocyanate; IBD, inflammatory bowel disease; ICAM-1, intercellular adhesion molecule 1; IL-1, interleukin 1; IVM, intravital microscopy; LFA-1, leucocyte function antigen $1 ; T N F-\alpha$, tumour necrosis factor $\alpha$; VLA-1, very late antigen 1 ; VCAM-1, vascular cell adhesion molecule 1; PBS, phosphate buffered saline. 
are oligomers, generally $15-25$ bases in length, designed to bind to mRNA by complementary base pairing (hybridisation). Local and intravenous administration of antisense oligonucleotides against the p65 subunit of nuclear factor $\mathrm{\kappa B}$ abrogated trinitrobenzene sulphonic acid induced colitis in mice. ${ }^{23}$ A murine specific ICAM-1 oligonucleotide (ISIS 3082) prevented dextran sulphate sodium induced colitis in mice in a dose dependent manner ${ }^{24}$ and a human specific ICAM-1 oligonucleotide (ISIS 2302) significantly lowered corticosteroid usage in a placebo controlled trial in patients with active steroid dependent Crohn's disease. ${ }^{25}$ In this study, newly designed chimeric oligonucleotides containing modified sugar residues and deoxynucleotides were used. Phosphorothioateoligonucleotides were modified by a methoxyethoxy group at the $2^{\prime}$ position of the ribose. These modifications exhibit an increase in potency, nuclease resistance, and duration of action compared with normal phosphorothioate oligonucleotides and lack immunostimulatory effects. ${ }^{26}{ }^{27}$

Using these modern oligonucleotides we investigated, by intravital microscopy (IVM), the effects of rat specific ICAM-1 (ISIS-17470) and VCAM-1 (ISIS 18155) second generation 2'-O-methoxyethyl chimeric oligonucleotides on leucocyteendothelial interactions in a rat model of IBD. For the first time we have shown that intravenous administration of antisense oligonucleotides against VCAM- 1 attenuates intestinal inflammation in an animal model of IBD. Furthermore, we demonstrated that intravenous application of ICAM-1 oligonucleotides was superior to subcutaneous application in controlling leucocyte endothelial adhesive interactions in this model of intestinal inflammation.

\section{METHODS}

\section{Induction of inflammation}

The experimental protocol was approved by the Animal Care Committee of the Regional Administration Muenster, Germany. Inbred male Sprague-Dawley rats (Charles River, Sulzfeld, Germany, 120-200 g) were single housed in standard laboratory cages with free access to water and food. Indomethacin (Sigma-Aldrich, Steinheim, Germany) at a dose of $7.5 \mathrm{mg} / \mathrm{kg}$ dissolved in $5 \% \mathrm{NaHCO}_{3}$ (Merck, Darmstadt, Germany) was injected subcutaneously over the left lower abdominal quadrant 48 and 24 hours prior to $\operatorname{IVM}^{28}$ (fig 1). Stool samples were tested daily for occult blood using hemoFEC (Boehringer Mannheim GmbH, Mannheim, Germany).

\section{Administration of oligonucleotides}

Oligonucleotides were obtained from ISIS Pharmaceuticals (Carlsbad, California, USA). ISIS 17470 is a rat specific 2'-O-methoxyethyl antisense oligonucleotide against ICAM-1 with the sequence 5'-TGTCCAGGTCACGAGTTCAC-3'. The underlined section carries the 2'-O-methoxyethyl modification. ISIS 18155 (5'-CGCGACCATCTTCACAGGCA-3') is a 2'-O-1-methoxyethyl antisense oligonucleotide which specifically targets VCAM- 1 in both rats and mice. As a scrambled

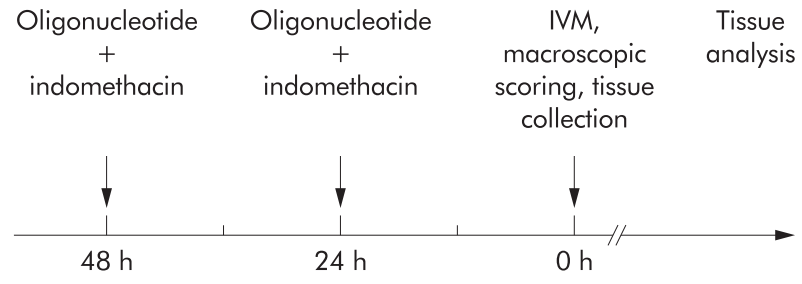

Figure 1 Experimental setting. Antisense oligonucleotides and indomethacin were administered at the same time 48 and 24 hours prior to intravital microscopy (IVM) indicating a prophylactic treatment schedule. Macroscopic scoring and sample collection for histological and immunohistochemical examination was performed immediately after IVM. control oligonucleotide, ISIS $18154 \quad\left(5^{\prime}\right.$ TCAAGCAGTGCCACCGATCC-3') was used. Parallel to subcutaneous indomethacin administration, oligonucleotides dissolved in phosphate buffered saline (PBS) were injected either subcutaneously or intravenously using a permanent central venous line (right jugular vein) 48 and 24 hours prior to IVM. A total of 110 rats were divided into 11 groups (each $\mathrm{n}=10$ ). Four control groups received scrambled control oligonucleotides (ISIS 181542 or $8 \mathrm{mg} / \mathrm{kg}$ intravenously) and indomethacin, indomethacin alone (disease control), or the indomethacin carrier $5 \% \mathrm{NaHCO}_{3}$ subcutaneously instead of indomethacin (healthy controls). Three treatment groups received indomethacin plus ICAM- 1 oligonucleotides at 1 and $2 \mathrm{mg} / \mathrm{kg}$ subcutaneously, or at $2 \mathrm{mg} / \mathrm{kg}$ intravenously. In the remaining four groups, indomethacin and VCAM-1 oligonucleotides were given at $1 \mathrm{mg} / \mathrm{kg}$ subcutaneously or at 2, 4, or 8 $\mathrm{mg} / \mathrm{kg}$ intravenously (fig $\mathrm{l}$ ). The initial dose of oligonucleotides, $1 \mathrm{mg} / \mathrm{kg}$ subcutaneously, was based on previous studies in a mouse model. ${ }^{24}$

\section{Intravital microscopy}

Under ether inhalation narcosis (Chinosol, Seelze, Germany) polyethylene catheters (internal diameter $0.58 \mathrm{~mm}$; Portex Ltd, Lythe, UK) were inserted into the right jugular vein and right carotid artery for administration of erythrocytes, contrast dyes, and pharmaceuticals as well as for monitoring arterial blood pressure and heart rate (Servomed, Hellige, Germany). Homologous erythrocytes were stained in vitro with fluorescein isothiocyanate (FITC; Sigma-Aldrich) before intravenous injection. Leucocytes were stained in vivo by intravenous injection of $17.5 \mu \mathrm{g} / 100 \mathrm{~g}$ rhodamine 6G (SigmaAldrich) immediately before IVM. ${ }^{29}$ Spontaneously breathing animals were placed in a supine position on a heating pad $\left(37^{\circ} \mathrm{C}\right)$ providing constant body temperature. The abdomen was opened by midline laparotomy and an ileum segment was gently positioned on an adjustable microscopy table. The exposed bowel was permanently superfused with $37^{\circ} \mathrm{C}$ warm saline and covered by cuprophane foil to avoid drying. Using an epiluminescence technique, leucocyte-endothelial cell interaction was visualised in 10 randomly selected ileal submucosal collecting venules (diameter $80-120 \mu \mathrm{m}$ ) after 15 minutes of equilibration, as previously described. ${ }^{30}$ A Zeiss fluorescence microscope was used, applying filters 450-490/ 515-565 nm for FITC and 510-560/>590 nm for rhodamine $6 \mathrm{G}$, respectively. Final magnification $\times 760$ on videoscreen was achieved by $16 \times / 0.5 \mathrm{~mm}$ water immersion objective (PlanNeofluar, Zeiss, Germany) and a mounted videocamera using 0.5 zoom (FK 6990-IQ, Pieper, Germany). Microscopic images were recorded for offline quantitative assessment of microcirculatory parameters using a computer assisted analysis system (analySIS; Soft Imaging System GmbH, Muenster, Germany). In each vessel the mean value (D) of five venular diameters was calculated. Central line erythrocyte velocity (V) was determined by calculating the mean velocity of five single frame to frame tracked red blood cells. Flow rate $\left(F_{t}\right)$ was calculated using the formula

$$
F_{t}=\left(\pi \times\left(D^{2} / 4\right) \times V \times t\right) / 10^{9}
$$

$(\mathrm{t}=$ time $) .^{30}$ Leucocytes were defined as adherent when attached to the vessel wall for at least 30 seconds and as rolling when moving with a velocity less than $2 / 5$ of that of erythrocytes at the centreline of the observed microvessel. ${ }^{29}$ Rolling and adherent cells were counted over a period of 30 seconds in a $100 \mu \mathrm{m}$ section of the vessel and given as numbers per 0.01 $\mathrm{mm}^{2}$ endothelial surface.

\section{Tissue analysis}

After IVM, animals were sacrificed by an overdose of potassium chloride injected into the right jugular vein. The 
entire gut was examined and the bowel was opened antimesenterically. Macroscopic appearance of inflammation was scored as described specifically for indomethacin induced ileitis. ${ }^{28}$ For histological grading, haematoxylin and eosin staining was performed on formalin fixed paraffin embedded sections taken from the IVM segments. Histological appearance was ranked in a blinded fashion using the modified score of Vilaseca and colleagues. ${ }^{31}$ Another IVM segment was snap frozen in liquid nitrogen for immunohistochemical studies. In some animals treated with VCAM-1 antisense oligonucleotides $8 \mathrm{mg} / \mathrm{kg}$ intravenously, immunohistological staining of VCAM-1 was performed using a standard peroxidase technique on cryostat sections. Endogenous peroxidase was blocked by incubation with 3\% hydrogen peroxide before antibody application. Goat polyclonal antibodies against rat VCAM-1 (sc-1504) were obtained from Santa Cruz Biotechnology, Inc. (California, USA). Secondary reagents were donkey antigoat IgG $(\mathrm{H}+\mathrm{L})$ and goat peroxidaseantiperoxidase (Jackson Immuno Research Laboratories, Inc., USA). ICAM-1 was detected immunohistochemically in some animals treated with ICAM-1 oligonucleotides $2 \mathrm{mg} / \mathrm{kg}$ intravenously using a murine monoclonal IgGl antibody versus rat ICAM-1 (1A29; Serotec Ltd, UK) and a polyclonal biotinylated goat antimouse IgG (PharMingen, San Diego, USA) as well as streptavidin-peroxidase (Dianova, Hamburg, Germany). Before antibody application, endogenous peroxidase and endogenous biotin were blocked by incubation with 3\% hydrogen peroxide and biotin-streptavidin, respectively. Negative controls were prepared by omission of the specific antibody. Slides were counterstained with Meyer's haematoxylin and made unrecognisable before examination.

\section{Statistics}

Results are given as mean (SD). Data were analysed using the Kruskal-Wallis test and an extended Fisher's exact test when appropriate; $p$ values $<0.05$ were considered to be significant. Rolling and adherent leucocytes and macroscopic scoring were correlated using linear correlation.

\section{RESULTS \\ Characterisation of leucocyte adhesion and inflammation in indomethacin induced ileitis}

The macroscopic aspect of small bowel inflammation typical of indomethacin induced enteritis in rats was a discontinuous transmural inflammation with multiple ulcerations and erosions of the small bowel with hyperaemic and haemorrhagic lesions. Administration of indomethacin caused a 5.3fold increase in leucocyte rolling $(27.8$ (5.3) $v 5.3(2.5) / 0.01$ $\mathrm{mm}^{2} ; \mathrm{p}<0.05$ ) (fig 2) and a 68-fold increase in leucocyte adhesion (14.0 (4.4) $\left.v 0.2(0.2) / 0.01 \mathrm{~mm}^{2} ; \mathrm{p}<0.05\right)$ (fig 3) compared with healthy controls. Macroscopic assessment of inflammation correlated closely with leucocyte rolling and also with leucocyte adherence (rolling leucocytes $r=0.73$, $\mathrm{p}<0.05$; adherent leucocytes $r=0.82, \mathrm{p}<0.05)$. Scrambled control oligonucleotides (ISIS 18155) $2 \mathrm{mg} / \mathrm{kg}$ intravenously did not reduce leucocyte rolling or adherence (24.9 (9.6)/0.01 $\mathrm{mm}^{2}$ and $15.5(4.5) / 0.01 \mathrm{~mm}^{2}$, respectively; NS $v$ indomethacin). Increasing the dose of scrambled control oligonucleotides up to $8 \mathrm{mg} / \mathrm{kg}$ intravenously also did not influence leucocyte adhesion $\left(25.2(6.1) / 0.01 \mathrm{~mm}^{2}\right.$ and $17.5(4) / 0.01 \mathrm{~mm}^{2}$, respectively; NS $v$ indomethacin or scrambled control $2 \mathrm{mg} / \mathrm{kg}$ intravenously). The oligonucleotide carrier PBS did not influence leucocyte-endothelial interactions. Scrambled control oligonucleotides $2 \mathrm{mg} / \mathrm{kg}$ intravenously showed the full macroscopic (4.2 (0.8) points (scrambled control) $v 4.5$ (0.7) (indomethacin)) and histological (5.1 (2) v 5.7 (2.4); NS) picture of indomethacin induced rat ileitis. This was confirmed by the higher dose of scrambled control oligonucleotides at $8 \mathrm{mg} / \mathrm{kg}$ intravenously (macroscopic $4.5(0.7)$ points and histological 6 (1.8) points). As in the indomethacin group, occult

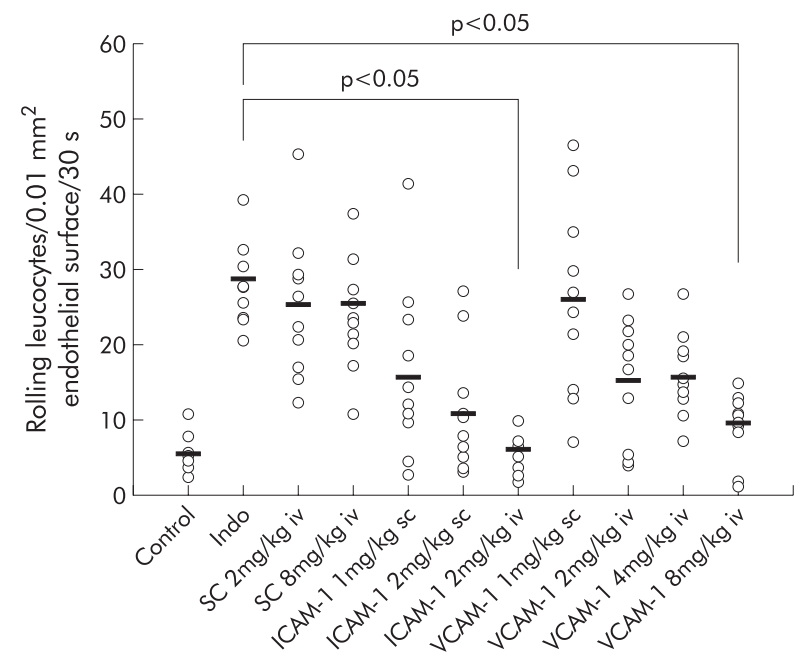

Figure 2 Rolling leucocytes 48 hours after the first application of indomethacin (Indo). The effect of antisense oligonucleotides on intestinal microcirculation observed by intravital microscopy. Two intravenous (iv) (but not subcutaneous (sc)) doses of $2 \mathrm{mg} / \mathrm{kg}$ intercellular adhesion molecule 1 (ICAM-1) antisense oligonucleotides and two iv doses of $8 \mathrm{mg} / \mathrm{kg}$ vascular cell adhesion molecule 1 (VCAM-1) antisense oligonucleotides reduced the number of rolling leucocytes significantly. Scatter plot with mean values indicated $(n=10)$. SC, scrambled control oligonucleotides.

faecal blood testing in all scrambled control oligonucleotide treated animals was $100 \%$ positive 48 hours after the first application of indomethacin. Erythrocyte velocity (mean 2250.8 (228.1) $\mu \mathrm{m} / \mathrm{s}$ ), blood flow (mean $1.1(0.2) \mu \mathrm{l} / \mathrm{min}$ ), and venular diameter (mean $101.0(7.5) \mu \mathrm{m})$ were not significantly different between the groups. Haematological investigations of peripheral blood showed 32.5\% neutrophils, $63.2 \%$ lymphocytes, $2.1 \%$ monocytes, and $1.4 \%$ eosinophils at 48 hours after the first application of indomethacin.

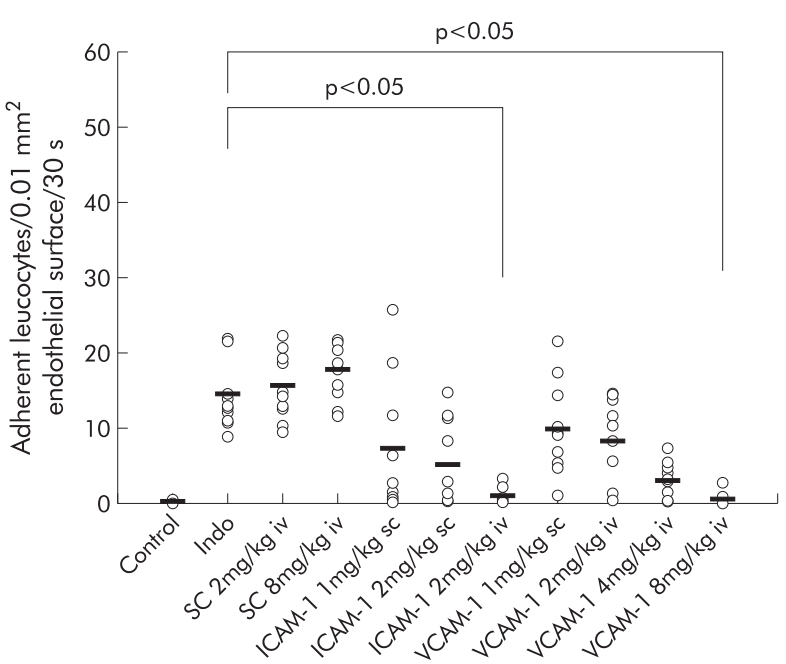

Figure 3 Adherent leucocytes 48 hours after the first application of indomethacin (Indo). The effect of antisense oligonucleotides on intestinal microcirculation observed by intravital microscopy. Two intravenous (iv) (but not subcutaneous (sc)) doses of $2 \mathrm{mg} / \mathrm{kg}$ intercellular adhesion molecule 1 (ICAM-1) antisense

oligonucleotides and two iv doses of $8 \mathrm{mg} / \mathrm{kg}$ vascular cell adhesion molecule 1 (VCAM-1) antisense oligonucleotides decreased the number of adherent leucocytes significantly. Scatter plot with mean values indicated $(n=10)$. SC, scrambled control oligonucleotides. 


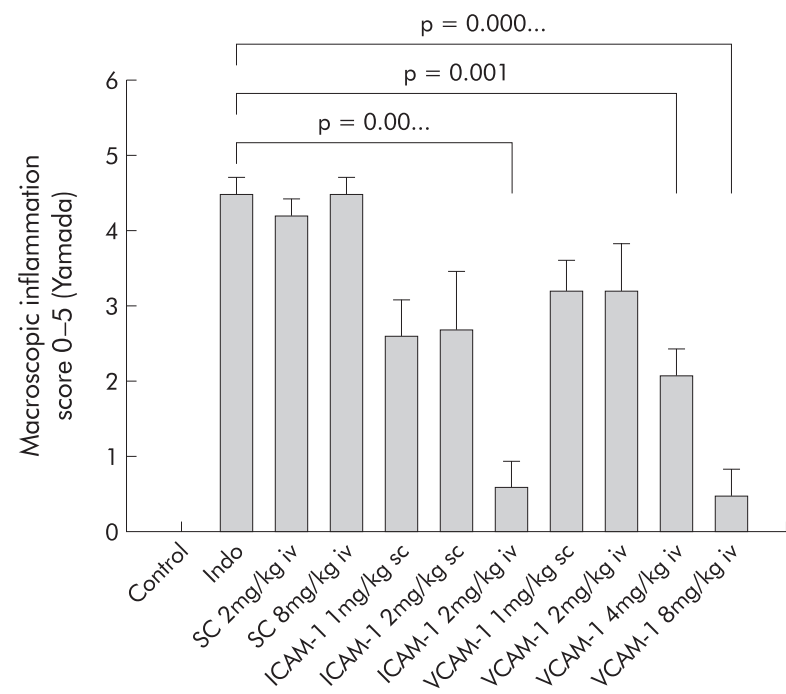

Figure 4 Macroscopic inflammation 48 hours after the first application of indomethacin (Indo). Two intravenous (iv) (but not subcutaneous (sc)) doses of $2 \mathrm{mg} / \mathrm{kg}$ intercellular adhesion molecule 1 (ICAM-1) antisense oligonucleotides and two iv doses of both $4 \mathrm{mg} / \mathrm{kg}$ and $8 \mathrm{mg} / \mathrm{kg}$ vascular cell adhesion molecule 1 (VCAM-1) antisense oligonucleotides significantly reduced macroscopic intestinal inflammation. Values are mean $(S E M)(n=\mid 0)$. SC, scrambled control oligonucleotides.

\section{Effect of ICAM-1 antisense oligonucleotides in intestinal inflammation}

Intravenous administration of ICAM- 1 oligonucleotides at a dose of $2 \mathrm{mg} / \mathrm{kg} 48$ and 24 hours prior to IVM caused a significant decrease in both leucocyte rolling (5.7 (2.4) v 27.8 (5.3)/ $\left.0.01 \mathrm{~mm}^{2} ; \mathrm{p}<0.05\right)$ and adherence $(0.8(1.1) \vee 14.0(4.4) / 0.01$ $\mathrm{mm}^{2} ; \mathrm{p}<0.05$ ) compared with inflamed controls (figs 2, 3). Injection of ICAM-1 oligonucleotides at $1 \mathrm{mg} / \mathrm{kg}$ or $2 \mathrm{mg} / \mathrm{kg}$ subcutaneously 48 and 24 hours prior to IVM failed to show a significant effect on leucocyte adhesion or on macroscopic or histological inflammation, although a tendency towards blunting cell adhesion was observed (figs 2-5). Macroscopic (0.6 (1.1) v 4.5 (0.7) points) (fig 4) and histological (0.6 (0.8) $v 5.7$ (2.4) points; $\mathrm{p}<0.001$ ) (fig 5) appearance of inflammation was significantly decreased by intravenous administration of ICAM-1 oligonucleotides $2 \mathrm{mg} / \mathrm{kg}$, and the appearance of occult faecal blood at the time of IVM was reduced to $30 \%$. In terms of macroscopic and histological inflammation, the effects of ICAM-1 oligonucleotides at $2 \mathrm{mg} / \mathrm{kg}$ were signifi-

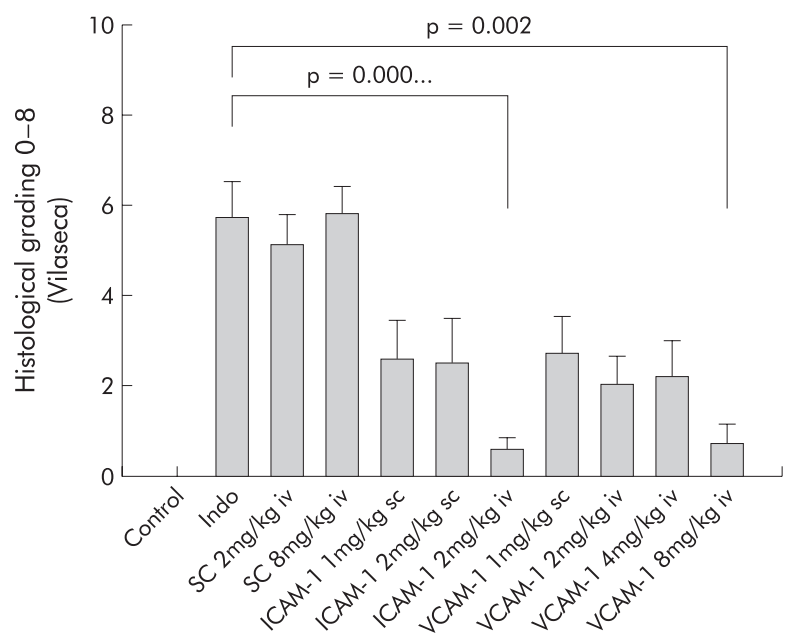

Figure 5 Histological inflammation 48 hours after the first application of indomethacin (Indo). Two intravenous (iv) (but not subcutaneous (sc)) doses of $2 \mathrm{mg} / \mathrm{kg}$ intercellular adhesion molecule 1 (ICAM-1) antisense oligonucleotides and two iv doses of $8 \mathrm{mg} / \mathrm{kg}$ vascular cell adhesion molecule 1 (VCAM-1) antisense

oligonucleotides significantly diminished histological intestinal inflammation. Values are mean $(S E M)(n=\mid 0)$. SC, scrambled control oligonucleotides.

cantly dependent on the route of administration, as intravenous and subcutaneous administration differed significantly $(p=0.035)$. Immunohistochemically, little constitutive ICAM-1 expression was detected in submucosal venules in healthy animals. Strong staining of ICAM-1 in submucosal venules in animals with indomethacin induced ileitis indicated that ICAM-1 expression was clearly upregulated compared with healthy controls. In animals treated with indomethacin and ICAM-1 oligonucleotides at a dose of $2 \mathrm{mg} / \mathrm{kg}$ intravenously, staining of ICAM-1 in submucosal venules was markedly reduced compared with diseased controls (fig 6).

\section{Effect of VCAM-1 antisense oligonucleotides in intestinal inflammation}

VCAM- 1 oligonucleotides at a dose of $8 \mathrm{mg} / \mathrm{kg}$ administered intravenously reduced both rolling $\left(9.2(4.4) / 0.01 \mathrm{~mm}^{2}\right.$; $\mathrm{p}<0.05)$ and adherent $\left(0.6(0.8) / 0.01 \mathrm{~mm}^{2} ; \mathrm{p}<0.05\right)$ leucocytes significantly (figs 2, 3). Treatment with VCAM-1 oligonucleotides $8 \mathrm{mg} / \mathrm{kg}$ intravenously significantly reduced
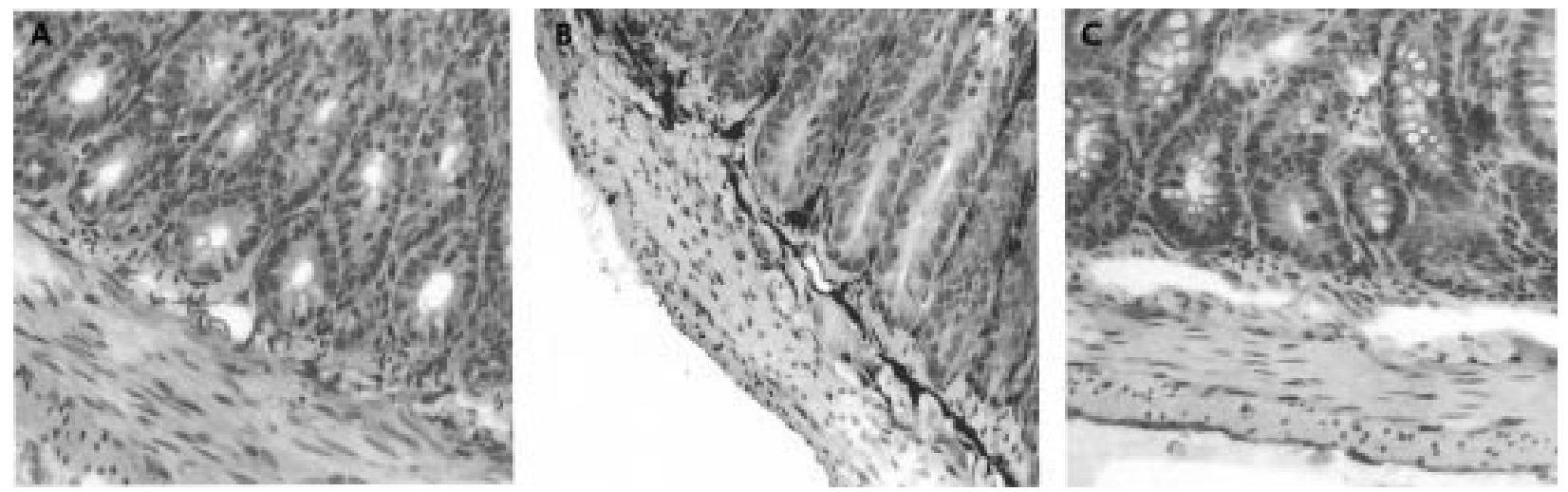

Figure 6 Intercellular adhesion molecule 1 (ICAM-1) expression in rat submucosal venules detected by immunohistochemistry using the peroxidase technique 48 hours after the first indomethacin application. (A) Frozen section of rat ileum from a healthy animal: small amounts of ICAM-1 are expressed constitutively in submucosal venules. (B) Frozen section of ileum from a rat with indomethacin induced ileitis: ICAM-1 expression is clearly upregulated in submucosal venules compared with healthy animals. (C) Frozen section of rat ileum from an animal treated with indomethacin and ICAM-1 antisense oligonucleotides at a dose of $2 \mathrm{mg} / \mathrm{kg}$ intravenously: staining of ICAM-1 in submucosal venules was markedly reduced compared with animals treated with indomethacin alone (original magnification $\times 200$ ). 

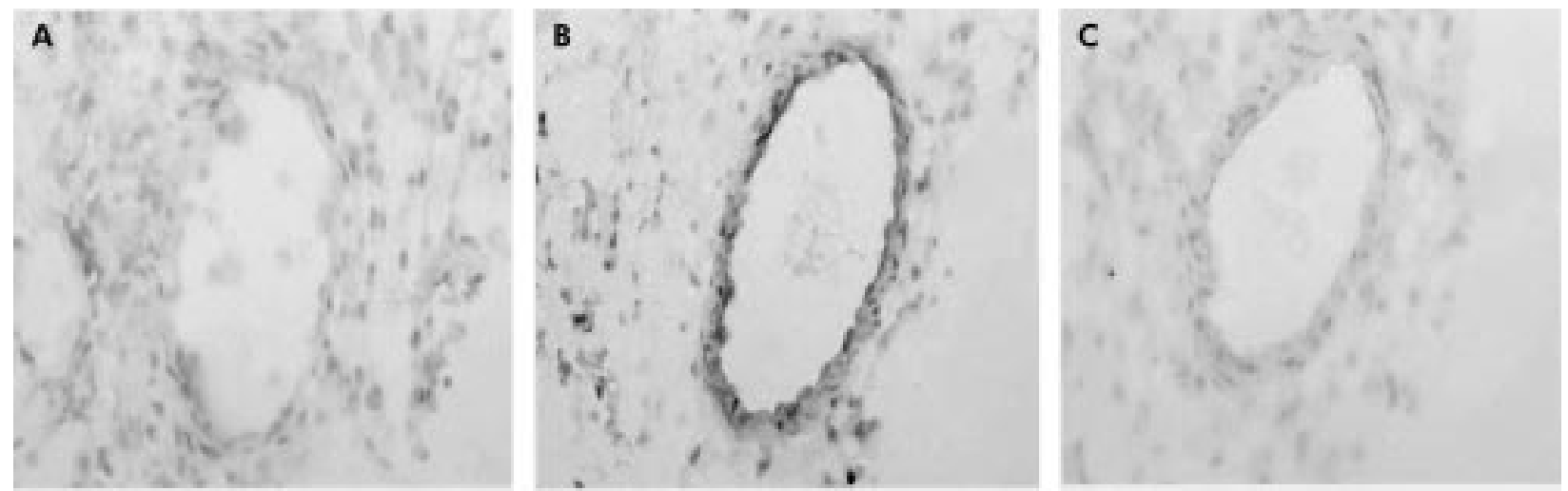

Figure 7 Immunohistochemical detection of vascular cell adhesion molecule 1 (VCAM-1) expression using a peroxidase technique in indomethacin induced ileitis in rats 48 hours after the first indomethacin application. (A) Frozen section of rat ileal mesenterium from a healthy animal: VCAM-1 is rarely expressed in mesenteric venules. (B) lleal mesenterium of an animal treated with indomethacin alone showing strong VCAM-1 staining in mesenteric venules. (C) Frozen section of rat ileal mesenterium from an animal treated with indomethacin and VCAM-1 antisense oligonucleotides at a dose of $8 \mathrm{mg} / \mathrm{kg}$ intravenously showing very little VCAM-1 staining in mesenteric venules (original magnification $\times 200$ ).

macroscopic (0.5 (1.1); p<0.001) (fig 4) and histological $(0.7$ (1.5); $\mathrm{p}=0.002$ ) inflammation (fig 5). Occult faecal blood was found at the time of IVM in $20 \%$ of animals treated with VCAM-1 oligonucleotides $8 \mathrm{mg} / \mathrm{kg}$ intravenously. Also, VCAM- 1 oligonucleotides $4 \mathrm{mg} / \mathrm{kg}$ intravenous attenuated indomethacin induced inflammation macroscopically $(p=0.001)$, although at this dose reduction of rolling and adherent leucocytes or histological inflammation was apparent but not significantly different. Immunohistochemically, VCAM-1 expression 48 hours after the first application of indomethacin was strong in diseased animals but rare in healthy controls. VCAM-1 was barely detectable in submucosal and mesenteric venules in animals treated with VCAM- 1 oligonucleotides dosed at $8 \mathrm{mg} / \mathrm{kg}$ intravenously (fig 7).

\section{DISCUSSION}

Since the understanding of the development of the intestinal cellular infiltrate has improved, prevention of cellular sequestration into intestinal tissues by blockade of adhesion molecules has become a major goal for therapeutic and prophylactic interventions. The use of oligonucleotides interfering with translation of mRNA of cell adhesion molecules seems promising to achieve this. Both 2'-O-methoxyethyl chimeric phosphorothioate antisense oligonucleotides (ISIS 17470 and ISIS 18155) designed to selectively inhibit ICAM-1 and VCAM-1 expression in rat cells reduced leucocyteendothelial interactions and prevented the development of indomethacin induced ileitis in a dose dependent manner, suggesting both adhesion molecules play a central role in the recruitment of leucocytes in this model.

The indomethacin model of acute intestinal inflammation is mainly driven by infiltrating neutrophils. This is well documented in morphological and immunohistochemical ${ }^{32}$ studies as well as in measurements of myeloperoxidase levels, which reflect neutrophil content in the tissue. ${ }^{28}{ }^{33}$ Although the pathogenesis of indomethacin induced intestinal inflammation remains unclear, increased mucosal permeability, bacterial translocation, ${ }^{28}$ microvascular disturbances, ${ }^{34}$ cyclooxygenase inhibition, ${ }^{28}{ }^{32}$ and enterohepatic circulation of indomethacin ${ }^{28}$ seem to play important roles in the initiation of the disease. At advanced stages of inflammation, macrophages $^{32}$ and lymphocytes are also present at sites of inflammation. Eosinophils or basophils have not been investigated in this model so far. Using immunohistochemical staining, we found marked enhancement of endothelial ICAM-1 and VCAM-1 expression in ileal submucosal and mesenteric venules in indomethacin induced ileitis compared with healthy controls. In a previous study using the dual labelled antibody method, we found clear upregulation of intestinal ICAM- 1 expression during the first 30 hours of inflammation but at later stages ICAM-1 seemed to be reduced. ${ }^{33}$ This could not be reproduced in our immunohistochemical studies in which ICAM-1 was still increased after 48 hours. However, expression of both adhesion molecules was clearly reduced after treatment with VCAM-1 or ICAM-1 oligonucleotides, indicating that amelioration of inflammation was mediated by inhibition of cell adhesion molecule transcription. A nonantisense mediated biological activity could be excluded by showing that scrambled control treated animals at both doses, and animals in the indomethacin group, were equally diseased. Therefore, it seems reasonable that infiltrating neutrophils adhere to the endothelium via both ICAM-1 and VCAM- 1 in this model.

The $\beta 2$ integrins LFA- 1 and MAC- 1 are expressed on the surface of neutrophils, monocytes, and natural killer cells. Lymphocytes however seem to express primarily LFA- $1,{ }^{3}$ enabling these inflammatory cells to bind to endothelial ICAM-1 and transmigrate into the tissue. Blockade of ICAM-1 by mononuclear antibodies ${ }^{1933} 35$ or antisense oligonucleotides ${ }^{24}$ reduces intestinal inflammation in various models. The ligand of VCAM-1, VLA-4, is expressed on lymphocytes ${ }^{3}$ but is also expressed on neutrophils under certain conditions. ${ }^{89}$ Interestingly, in a state of inflammation, the $\alpha 4 \beta 1$ /VCAM-1 pathway of neutrophil recruitment seems to develop increasing importance. $^{910}$ Our observation that inhibiting VCAM-1 expression by means of antisense oligonucleotides blocks neutrophil recruitment can be explained by these findings. With regard to prophylactic treatment in our model, inhibition of lymphocyte recruitment in the later stages of this model by VCAM-1 antisense oligonucleotides may be of minor importance. In other models of IBD with a more chronic character, monoclonal antibodies versus VCAM-1 were highly effective in the control of intestinal inflammation. ${ }^{196}$ VCAM-1 expression has been shown to be markedly upregulated in these models and was even well correlated with myeloperoxidase levels. ${ }^{19}$ The role of ICAM- 1 and VCAM- 1 in firm adherence of leucocytes to endothelial cells is well established but both adhesion molecules also support leucocyte rolling. IVM studies demonstrate that ICAM-1 is required for optimal P- and L-selectin mediated rolling of leucocytes in inflamed vessels ${ }^{37}$ while blockade of VCAM-1 results in increased rolling velocity of mononuclear cells. ${ }^{38}$ The observation that a certain antisense oligonucleotide at a high enough dose shows leucocyte adhesion comparable with that seen in healthy controls does not imply that rolling or sticking was completely abrogated. Rather it has been shown that ICAM-1 and 
VCAM-1, respectively, were crucial for the development of intestinal inflammation in this model. The reduction in leucocyte adhesion may also have been a consequence of a general decrease in leucocyte and endothelial activation in oligonucleotide treated animals. This might have been caused by interrupting the amplification of the inflammatory response, which is normally generated by chemotactic reagents such as IL-8 or MIP- 1 released by transmigrated inflammatory cells. Apparently, both LFA-1/ICAM-1 and VLA-4/VCAM-1 interaction play a central role in this concert. Reducing the adhesion cascade to a certain threshold may also disturb alternative pathways of leucocyte activation and recruitment in this model.

When comparing ICAM-1 and VCAM-1 oligonucleotides given at the same doses and by the same administration route, ICAM-l seemed to be more effective than VCAM-1. Nevertheless, it must be considered that in animal models VCAM-1 upregulation under inflammatory conditions is stronger than ICAM- $\mathrm{l}^{19}$ and therefore VCAM-1 oligonucleotides had to be given at a four times higher dose to produce similar effects. This may be a consequence of the different epitope targeted but may also be due to the different structures of the oligonucleotides, underlining the need for specific testing of pharmacokinetics of each individual oligonucleotide before application. Future studies might examine the beneficial effects of a combination of ICAM- 1 and VCAM-1 oligonucleotides in terms of dose reduction and additional decrease in inflammation. Macroscopic inflammation was significantly reduced by VCAM-1 oligonucleotides at a dose of $4 \mathrm{mg} / \mathrm{kg}$ intravenously although leucocyte adhesion and histological inflammation were not. This may be explained by previous studies showing altered expression of cell adhesion molecules in uninvolved gut; thus adhesion of circulating leucocytes to the mucosal endothelium may precede macroscopic visible inflammation. ${ }^{39}$ Influx of inflammatory cells into the tissue is a dynamic process which is constantly perpetuated during inflammation. Therefore, patients with established IBD may benefit from oligonucleotides against ICAM-1 or VCAM-1. As VCAM-1 expression is very rare in healthy subjects but strongly upregulated under inflammatory conditions, VCAM-1 seems to be a more specific drug target compared with ICAM-1 which is constitutively expressed and upregulated to a lower extent. ${ }^{19}$ However, VCAM-1 has not been shown to be upregulated in biopsy specimens from patients with IBD. ${ }^{12}{ }^{16}{ }^{17}$ As plasma levels of sVCAM-1 were increased in these patients ${ }^{14}{ }^{15}$ and monoclonal antibodies versus the $\alpha 4$ integrin showed promising effects in reduction of the disease activity index in patients with active Crohn's disease, ${ }^{40}$ a VCAM-1/ $\alpha 4$ interaction may also be important in human intestinal inflammation. Given recurrent cellular infiltration at the sites of surgical anastomoses in up to $50 \%$ of cases even three months after surgery, ${ }^{41}$ permanent local control of cellular sequestration with the help of intestinal oligonucleotides against ICAM-1 or VCAM-1 seems reasonable. To this aim, oligonucleotides seem to be preferable to monoclonal antibodies because the efficiency of the latter is impaired by immunoneutralisation, even in the case of chimeric monoclonal antibodies (approximately 75\% human) and humanised monoclonal antibodies (approximately 95\% human). ${ }^{42}$ Further studies should aim to design pharmacological preparations which provide high levels of local intestinal oligonucleotides for long lasting suppression of cell adhesion and intestinal inflammation in IBD.

\section{ACKNOWLEDGEMENTS}

Supported by a research grant from the Crohn's Disease and Ulcerative Colitis Foundation of Germany DCCV eV and a research grant from the Westphalian Wilhelms-University Muenster (IMF SC-1-1-II (96/5)). Dr CF Bennett is employed at ISIS Pharmaceuticals, Carlsbad, CA 92008, USA.

\section{Authors' affiliations}

E Rijckent, C F Krieglsteint, C Anthoni, M G Laukoetter,

R Mennigen, Hu Spiegel, N Senninger, G Schuermann, Department

of General Surgery, Westphalian Wilhelms-University, Muenster,

Germany

C F Bennett, ISIS Pharmaceuticals, Carlsbad, CA 92008, USA

†E Rijcken and C F Krieglstein contributed equally to this work.

\section{REFERENCES}

1 Dustin ML, Springer TA. Role of lymphocyte adhesion receptors in transient interactions and cell locomotion. Annu Rev Immunol $1991 ; 9: 27-66$.

2 Shimizu Y, Newman W, Tanaka Y, et al. Lymphocyte interactions with endothelial cells. Immunol Today 1992;13:106-12.

3 Panés J, Granger DN. Leukocyte-endothelial cell interactions: molecular mechanisms and implications in gastrointestinal disease. Gastroenterology 1998;114:1066-90.

4 Myers CL, Wertheimer SJ, Schembri-King J, et al. Induction of ICAM-1 by TNF- $\alpha$, IL-1 $\beta$, and LPS in human endothelial cells after downregulation of PKC. Am J Physiol 1992;263:C767-72.

5 Haraldsen G, Kvale D, Lien B, et al. Cytokine-regulated expression of E-selectin, intercellular adhesion molecule-1 (ICAM-1), and vascular cell adhesion molecule-1 (VCAM-1) in human intestinal microvascular endothelial cells. J Immunol 1996;156:2558-65.

6 Anderson DC. The role of $\beta 2$ integrins and intercellular adhesion molecule type 1 in inflammation. In: Granger DN, Schmid-Schoenbein GW, eds. Physiology and pathophysiology of leukocyte adhesion. New York: Oxford University Press, 1995:3-42.

7 Kubes P, Niu XF, Smith CW, et al. A novel beta 1-dependent adhesion pathway on neutrophils: a mechanism invoked by dihydrocytochalasin $B$ or endothelial transmigration. FASEB J 1995;9:1103-11.

8 Henderson RB, Lim LHK, Tessier PA, et al. The use of lymphocyte function-associated antigen (LFA)-1-deficient mice to determine the role of LFA-1, Mac-1, and $\alpha 4$ integrin in the inflammatory response of neutrophils. J Exp Med 2001;194:219-26.

9 Johnston B, Kubes P. The $\alpha 4$-integrin: an alternative pathway for neutrophil recruitement? Immunol Today 1999;20:545-50.

10 Ibbotson GC, Doig C, Kaur J, et al. Functional $\alpha 4$-integrin: a newly identified pathway of neutrophil recruitement in critically ill septic patients. Nat Med 2001;7:465-470.

11 Malizia G, Calabrese A, Cottone M, et al. Expression of leukocyte adhesion molecules by mononuclear phagocytes in inflammatory bowel disease. Gastroenterology 1991;100:150-9.

12 Nakamura S, Ohtani H, Watanabe $Y$, et al. In situ expression of the cell adhesion molecules in inflammatory bowel disease. Lab Invest 1993;69:77-85.

13 Bernstein CN, Sargent M, Gallatin WM. $\beta 2$ Integrin/ICAM expression in Crohn's disease. Clin Immunol Immunopathol 1998;86: 147-60.

14 Jones SC, Banks RE, Haidar A, et al. Adhesion molecules in inflammatory bowel disease. Gut 1995;36:724-30.

15 Göke M, Hoffmann JC, Evers J, et al. Elevated serum concentrations of soluble selectin and immunoglobulin type adhesion molecules in patients wih inflammatory bowel disease. J Gastroenterol 1997;32:480-6.

16 Oshitani N, Campbell A, Bloom S, et al. Adhesion molecule expression on vascular endothelium and nitroblue tetrazolium reducing activity in human colonic mucosa. Scand J Gastroenterol 1995;30:915-20.

17 Koizumi M, King N, Lobb R, et al. Expression of vascular adhesion molecules in inflammatory bowel disease. Gastroenterology 1992; 103:840-7

18 Henninger DD, Panés J, Eppihimer $M$, et al. Cytokine induced VCAM-1 and ICAM-1 expression in different organs of the mouse. J Immunol 1997:158:1825-32

19 Sans M, Panés J, Ardite E, et al. VCAM-1 and ICAM-1 mediate leukocyte-endothelial cell adhesion in rat experimental colitis. Gastroenterology 1999;116:874-83.

20 Crooke ST. Therapeutic applications of oligonucleiotides. Biotechnology (NY) 1992;10:882-6

21 Bennett CF, Condon TP, Grimm S, et al. Inhibition of endothelial cell adhesion molecule expression with antisense oligonucleotides. J Immunol 1994; 152:3530-40.

22 Hartmann G Bidlingmaier M, Eigler A, et al. Cytokines and therapeutic oligonucleotides. Cytokines Cell Mol Ther 1997;3:247-56.

23 Neurath MF, Pettersson S, Meyer zum Buschenfelde KH, et al. Loca administration of antisense phosphorothioate oligonucleotides to the p65 subunit of NF-kappa B abrogates established experimental colitis in mice. Nat Med 1996;2:998-1004.

24 Bennett CF, Kornbrust D, Henry S, et al. An ICAM-1 antisense oligonucleotide prevents and reverses dextran sulfate sodium-induced colitis in mice. J Pharmacol Exp Ther 1997;280:988-1000.

25 Yacyshyn BR, Bowen-Yacyshyn MB, Jewell L, et al. A placebo-controlled trial of ICAM-1 antisense oligonucleotide in the treatment of Crohn's disease. Gastroenterology 1998;114:1133-42.

26 Baker BF, Lot SS, Condon TP, et al. 2'-O-(2-methoxy)ethyl-modified anti-intercellular adhesion molecule 1 (ICAM-1) oligonucleotides selectively increase the ICAM-1 mRNA level and inhibit formation of the ICAM-1 translation initiation complex in human umbilical vein endothelia cells. J Biol Chem 1997;272: 1 1994-2000. 
27 Glover JM, Leeds JM, Mant TGK, et al. Phase I safety and pharmacokinetic profile of an intercellular adhesion molecule-1 antisense oligodeoxynucleotide (ISIS 2302). J Pharmacol Exp Ther 1997; 282:1173-80

28 Yamada T, Deitch E, Specian RD et al. Mechanisms of acute and chronic intestinal inflammation induced by indomethacin. Inflammation 1993;17:641-62.

29 Gonzalez AP, Sepulveda S, Massberg S, et al. In vivo fluorescence microscopy for the assessment of microvascular reperfusion injury in small bowel transplants in rats. Transplantation 1994:58:403-8.

30 Krieglstein CF, Anthoni C, Laukoetter MG, et al. Effect of anti-CD $11 \mathrm{~b}$ $\left(\alpha^{M}-M A C-1\right)$ and anti-CD54 (ICAM-1) monoclonal antibodies on indomethacin induced chronic ileitis in rats. Int J Colorectal Dis 1999;14:219-23.

31 Vilaseca J, Salas A, Guarner F, et al. Participation of thromboxane and other eicosanoids synthesis in the course of experimental inflammatory colitis. Gastroenterology 1990;98:269-77.

32 Nygard G, Anthony A, Piasecki C, et al. Acute indomethacin-induced jejunal injury in the rat: early morphological and biochemical changes. Gastroenterology 1994:106:567-75.

33 Krieglstein CF, Salter JW, Cerwinka WH, et al. Role of intercellular adhesion molecule 1 in indomethacin induced ileitis. Biochem Biophys Res Commun 2001;282:635-42.

34 Kelly DA, Piasecki C, Anthony A, et al. Focal reduction of villuos blood flow in early indomethacin enteropathy: adynamic vascular study in the rat. Gut 1998;42:366-73.
35 Taniguchi T, Tsukada $\mathrm{H}$, Nakamura $\mathrm{H}$, et al. Effects of the anti-ICAMmonoclonal antibody on dextran sodium sulphate-induced colitis in rats. $J$ Gastroenterol Hepatol 1998; 13:945-9.

36 Soriano A, Salas A, Salas A, et al. VCAM-1, but not ICAM-1 or MAdCAM-1, immunoblockade ameliorates DSS-induced colitis in mice. Lab Invest 2000;80: 1541-51.

37 Steeber DA, Campbell MA, Basit A, et al. Optimal selectin-mediated rolling of leukoyctes during inflammation in vivo requires intercellular adhesion molecule-1 expression. Proc Natl Acad Sci U S A 1998;95:7562-7

38 Ramos CL, Huo Y, Jung U, et al. Direct demonstration of P-selectin and VCAM-1-dependent mononuclear cell rolling in early artherosclerotic lesions of apolipoprotein E-deficient mice. Circ Res 1999;84:1237-44.

39 Schuermann G, Aber-Bishop AE, Facer P, et al. Altered expression of cell adhesion molecules in uninvolved gut in inflammatory bowel disease. Clin Exp Immunol 1993;94:341-7.

40 Gordon FH, Lai CWY, Hamilton Ml, et al. A randomized placebo-controlled trial of a humanized monoclonal antibody to $\alpha 4$ integrin in active crohn's disease. Gastroenterology 2001;121:268-74.

41 Rutgeerts P. Hiele M, Geboes K, et al. Controlled trial of metronidazole treatment for prevention of Crohn's recurrence after ileal resection. Gastroenterology 1995; 108:1617-21.

42 Sandborn WJ, Hanauer SB. Anti-tumor necrosis factor therapy for inflammatory bowel disease: a review of agents, pharmacology, clinical results, and safety. Inflamm Bowel Dis 1999;5:119-33.

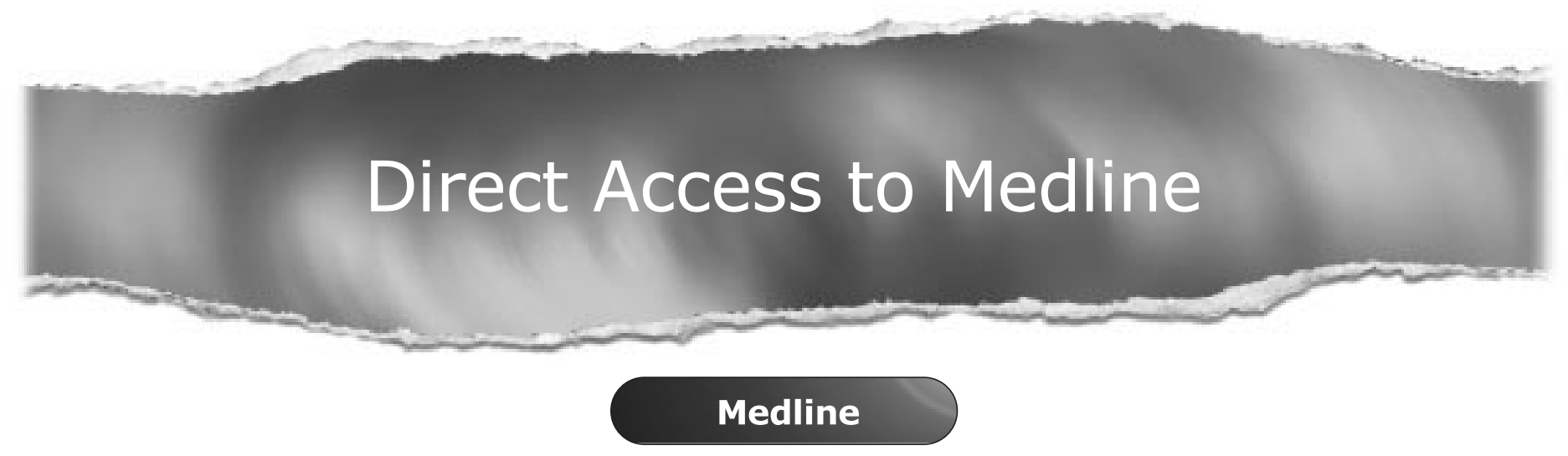

Link to Medline from the homepage and get straight into the National Library of Medicine's premier bibliographic database. Medline allows you to search across 9 million records of bibliographic citations and author abstracts from approximately 3,900 current biomedical journals.

www.gutjinl.com 